apex; and the apex of the opposite lung contained also much crude tribercle and some few vomicx.

The liver was much enlarged, and projected up considerably into the thorax on the left side. It weighed 9 lbs. 2 oz., and was very hard and firm in texture. It, as well as the kidneys, contained a good quantity of fat. The spleen was enlarged.

ReMarks. In this case great difficulty was experienced in coming to a correct diagnosis as to the cause of the pulsation. The opinion expressed during life was, however, justified by the past mortem appearances; and it will be well to bear in mind the occurrence occasionally of these displacements of the heart, in patients whose lungs are bound down by old disease; in consequence of which the pulmonary artery, dilated probably from the old retardation of the pulmonic circulation, is brought up directly against the wall of the chest, and occasions a pulsation which might almost be mistaken for that of anearism.

\section{Oriainal Commumations.}

\section{CASE OF PANCREATIC CYST.}

\section{By Joshua Parsons, Esq., Beckington, Somerset.}

[Read before the Bath and Bristol Branch, April 30th, 1857.]

Mrs. W., aged 60 , the wife of a clergyman, a lady of good general health and great activity, the mother of ten children, had been for many years subject to occasional attacks of spasm of the stomach, which had latterly increased in frequency and severity. In the month of April 1853, she took a journey into a distant county, and while there was seized with an unusually severe attack of spasm, resisting the usual means of relief, and apparently followed by gastric inflammation. This inflammation was treated, under the care of the medical friend in whose house she was visiting, by leeching and mercurials, carried to the extent of slight ptyalism. She slowly improved so far as to be able to journey homewards ; after which, she came immediately under my care.

At this time, the symptoms were those of chronic gastritis; and as such, they proceeded without much change, sometimes alleviated by treatment, but with loss of flesh and strength, till the month of August.

One day, at the beginning of that month, she called my attention to a tumour which she had discovered in the epirastrium. How long it had existed there, we cannot tell. I had frequently made manual examinations of that region, but, owing to the great tenderness, had hesitated to make such deep pressure as would perhaps have discovered its existence earlier. The swelling was now of about the size of a small orange, slightly moveable, situated immediately in front of the spine, and evi dently behind the stomach, as the flatus in that organ could be felt by the hand rolling about in front of it. The swelling increased steadily for about a fortnight, till it had reached the size of a cricket-ball, when one morning the patient told me with great satisfaction, that it had entirely disappeared; but that she had been suffering all night from diarrhoa of a pecu liar character. The discharges were entirely fluid, of a glairy consistence, dirty white colour, with a few flakes, and of an odour which immediately struck me as being salivary. It was precisely similar to that produced by spitting on hot iron. Some relief of the gastric symptoms followed this discharge but it was only temporary. The tumour soon reappeared, and attained its former dimensions, but never in that region much exceeded them. Symptoms of kjdney irritation now appeared, with much pain and tenderness in the left lumbar and iliac regions; and, after a few weeks, a tumour appeared immedi ately above the anterior superior or spinous process of the ilium which presented the same feel as the former one, and was plainly continuous with it; increased tension of the one being produced by pressure on the other.

For many weeks no remarkable change occurred: the last formed tumour enlarged slowly. There were great suffering and increasing emaciation and debility. The patient removed to some distance, so that I could only see her at long intervals and I am greatly indebted for the closing history of the case and the particulars of the post mortem examination, to my friend Mr. Chitty, of Mere.

At the beginning of February 1854 , nine months from the com mencement of the illness, a return of the diarrhœa occurred, with subsidence of the swellings, and relief of the pain. This lasted for two days, and then the tumour filled somewhat suddenly again. In ten days, the diarrhœea returned, with copious vomiting; this time attended with discharge of much dark grumous blood, both from the bowels and stomach; and the patient was evidently sinking rapidly.

On February 25 th, I visited her, and found her greatly reduced and extremely emaciated; but the tumours, though perceptible, were flaccid, and had ceased to give the sense of fluctuation. While I was sitting in her room, she suddenly stretched out her arms, desiring to be lifted up in bed, became immediately unconscious, with a blanched face, cold perspirations, and imperceptible pulse, and died in a short time, with all the symptoms of internal hæmorrhage.

The following is the account of post mortern appearances, kindly supplied to me by Mr. Chitty. There was no peritonitis. The intestinal canal and the other abdominal viscera were of a dark leaden hue. The stomach was small and contracted; its large curvature was elevated; the smaller was bound down tightly by adhesions, with what remained of the pancreas, to the spine, and this firm adhesion extended by the spleen and left kidney to the left iliac fossa. By these adhesions a canal was formed, in which was the morbid pancreas, consisting apparently of a long cystic tube, extending from the duodenum to the left hypochondrium, fully six inches in length. Behind the descending colon it become a cavity, of the size of the fist, containing throughout a dark grumous fluid, like decomposed blood. The walls of the cyst were of cartilaginous hardness, and lined with a dark brown substance. There was also within the cyst an extravasation of recent blood. The tumour was immediately opposite to, and adherent with, the colon, which gut was disorganised and soft, but not ulcerated; nor could I discover any communication between it and the cyst. The left kidney was softened and disorganised, but contained no trace of calculous disease. The spleen was small, soft, and black. The left pleura contained much serum, and the lung was small, collapsed, and covered with a layer of fibrine. The other organs of the body appeared healthy, though greatly wasted.

Remarks. So far as my limited opportunities of consulting medical literature extend, the case now detailed appears to be a rare one. Its pathological history is interesting, but, unfortunately, lacks a commencement. Such a cyst as I have described, appears to find its analogue in ranula of the sublingual and submaxillary glonds; but amidst the displacement and disorganisation which had confused all the parts, it was, of course, impossible to ascertain the condition of the pancreatic ducts at the time of the post mortem examination. The first morbid change was retention of the pancreatic secretion; and it is possible, though of course I only offer it as a conjecture, that such a retention might have been carsed by the gastric inflammation which succeeded the primary attack of spasm, extending either to the ducts themselves or the investing parts. The salivary organs being at this conjuncture stimulated to extra secretion, an amount of fluid sufficient to prodnce dilatation might the more readily have accumulated. If we suppose a cyst to have thus originated, its subsequent progress and relation to the symptoms seem very clear. A temporary abatement of its progress occurred; when, about a fortnight after its first discovery, the contents were evacuated through the bowels. This discharge probably took place throuch the natural outlets; but as in ranula, if the salivary ducts are not enlarged or kept patent by artificial means, the accumulation soon recurs, so there was a speedy return of the symptoms. The cyst now enlarging, and prevented from spreading to the right side by the comparatively firm structures at the root of the liver, made itself a way behind the spleen and kidney to the left hypochondrium, producing great renal disturbance, perhaps aggravated by the presence of a small calculus that was voided about this time (September). For four months, no remarkable change, except progressive enlargement, occurred. The cyst had come into contact with the descending colon, and had involved the gut in the destructive irritation of its presence, and now another discharge of its contents took place. The speedy return to the original dimensions of the tumour must, I think, be attributed to extravasation of blood, thus accounting for the great loss of vital power by which it was accompanied. This blood, retained long enough to become decomposed, furnished the grumous discharges of the last attack of diarrhœa, as well as the similar matter found in the sac after death, while the loss of a second quantity of blood there found undecomposed, sufficed at last to extinguish the flickering powers of life.

Though the nature of the disease in this case seems so clear 
under the light of the scalpel, it was by no means so during life; and various were the opinions and conjectures offered by several medical men who saw, or were consulted on, the case. To my mind, the character of the discharge attending the first subsidence of the tumour was strong evidence of the correctness of that diagnosis which dissection confirmed.

I can offer no suggestions respecting the curative treatment of this malady; and, indeed, it seems difficult to conceive how any such could be applied : but I will hazard one remark that, while it bears upon the physiological use of the pancreatic fluid, might also suggest a means of relieving the sufferings attending such a melancholy case. During the whole course of this case, there was never any uniform or persistent disturbance of the bowels. There were the three attacks of diarrhœa already mentioned, and on one or two occasions some disorder caused by manifest error of diet; but in general the bowels were neither costive nor re. laxed, nor were the appearance or consistence of the secretions unnatural. On the other hand, the stomach was in a perpetual state of severe irritation, bordering on, if it did not amount to, actual inflammation; and this, it appears to me, arose, not only from the interference of the tumour behind it, but from the acrid properties of its own undiluted secretions; and if so, some relief might be afforded by the exhibition, during the times when the stomach is not actively employed in digestion, of large doses of bland mucilaginous fluids, holding small quantities of soda in solution. In accordance with this view, it was found that in this case fresh made whey, cold and frequently sipped, alleviated the sickness and pain more than any other article of diet or medicine.

\section{NEW OPERATION FOR PHIMOSIS.}

By A. G. Walter, Surgeon, Pittsburgh, Pennsylvania, U. S. THE various methods for the relief of phimosis, which have been introduced and practised from the oldest times of surgery to the present, though answering the purpose intended, cannot fail to impress upon the mind of the surgeon of the present day the ardent desire to have an operation substituted, which will be free from the objection of producing mutilation or deformity. It behoves the conservative surgeon, when called on to remove deformities of the human body, or to relieve obstructions in the different organs, to save any and every healthy part of the human system, and to remove the infirmities, either congenital or acquired, with the least expense to the body; for Nature, in her wisdom, has not created the smallest particle of human organism but for some especial purpose. The healthy body is perfect in all its parts; nothing superfluous can be found about it. By some mysterious agencies, however, Nature at times departs from the customary path during formation; she is arrested in her developments of parts of the human system; and deformities, defects, malformations, and obstructions, are the consequence. To relieve these, man's genius has been taxed; improvements of the various means have followed each other; but it was left to the present age, enlightened and ennobled by the blessings of conservative surgery, to make surgeons pause, reflect, and study Nature's inexhaustible powers, and to send them forth as restorers, and not as mutilators.

In congenital phimosis, and frequently also in the acquired form, we find the deformity to be produced by the tightness of the frænum ; the constriction being seated in the internal layer of the prepuce, the external one being free from fault, and therefore not requiring to be interfered with. To overcome this ab. normal formation of the prepuce, circumcision, division of both layers of the prepuce on its dorsal or inferior aspect, are practised. These methods, no doubt, relieve the defect; but they also produce artificial deformity of the member. By the practice which $I$ intend to offer to the consideration of the profession, these opprobria chirurgorum are avoided, and the member is restored to its natural and healthy condition.

The penis is held firmly and horizontally by the fingers of an assistant, which grasp it near its root, drawing at the same time the prepuce backwards over the body of the organ towards the pubes as much as possible; a fine grooved director, with a blun point (its groove pointing downwards) is then inserted between the prepuce and glans penis, close to the right side of the frænum, and is pushed on till it is arrested by the reflexion of the inner layer at the corona glandis. A fine bladed scalpel is next passed into the groove of the director, its cutting edge looking downwards; and both layers of the prepuce at the orifice are divided. The knife being pushed on in the groove, while the assistant draws the prepuce farther back, the internal layer is slit up close to the frænum, as far as the corona glandis. A notch only being made in the external layer, the retraction of the prepuce is effected by the complete division of the inner layer. Some tension, however, of the inner layer, still remaining, the director is passed close to the other side of the frænum, between the glans penis and the inner layer of the prepuce; and upon it on this side, too, the inner layer is cut through, close to the frænum, up to the corona glandis: the external layer, being drawn far back, is beyond the reach of the knife. By directing the incision in this manner, we find the frænum and glans penis fully exposed, and a cut on each side of the frænum, in the internal state of the prepuce, running in an outward direction from each side of the frænum. After arresting the bleeding, which is generally trifling, both lips of the wound along the frænum are united over it with two or three stitches by a fine needle and thread. This done, the wound on each side of the frænum, in the internal layer of the prepuce, is accurately united by several sutures to the external or dermal layer. All threads being cut off, and the glans penis covered with a fine piece of oiled muslin, the nrepuce is returned to its natural position over the head of the jenis. Adhesion between the cut surfaces of the internal layer of the prepuce and the frænum being thus prevented by accurately adjusted sutures, there can be no apprehension of a return of the deformity. Beyond an cedematous swelling of the prepuce-the consequence of the operation-lasting for a few days, and easily relieved by lukewarm fomentations of chamomile and Goulard's lotion, while the patient is at rest, no untoward symptoms will follow. The sutures, being very fine, need not be removed till all swelling of the prepuce has subsided, which will take place in the course of a week or two.

By operating in this manner, the prepuce retains its natural appearance; no scar is visible on its external face; it allows of easy retraction; and continues to cover the glans, as nature had designed.

Having repeatedly practised this method both for acquired and congenital phimosis, I feel anxious that other surgeons should give it that consideration which it merits on account of its simplicity and certainty of cure, leaving no trace whatever of either deformity or mutilation.

\section{INTERNAL STRANGULATION OF INTESTINE RAPIDLY FATAL.}

By Henry Ewen, Esq., Long Sutton, Lincolnshire.

ON August 18th, 1856, L. F., a girl, aged 14, complained at noon of being unwell. She had pain in the left side, but took her dinner as usual; she walked out with the children of her charge for an hour in the afternoon, but on her return complained of being exceedingly unwell, the pain in the left side having increased; she could not take her tea. She went to bed at 10 P.M., when copious vomiting came on, with great increase of pain in her left side. The vomiting was not stercoraceous. There was no hiccough. Fomentations and other domestic remedies were used with partial relief, but she was restless and in great pain all night; there was frequent and urgent desire to pass urine, but very little was voided. At 6 A.M. on Aug.19th, complete collapse had come on. She had never menstruated. I was sent for about 7 in the morning, and reached the house about 8 ; she was then dead.

An inspection of the body was made seven hours after death. She was a short, delicate young woman. The abdomen was hard and distended; there were about two pints of bloody serum affused into the cavity. The whole of the jejunum and ileum had passed through an aperture in the mesentery behind the middle third of the duodenum; the last portion of the ileum had drawn upwards the cæcum with the vermiform appendix, which were seen just below the edge of the liver. The cæcum and ascending colon were much distended with gas. All that portion of intestine which had passed through the aperture in the mesentery was of a dark chocolate colour, and much distended with gas, and there were many patches of blood extravasated in the corresponding portions of the mesentery. The stomach was healthy, and contained about a pint of milky fluid. The bladder was empty. The lungs with their pleuræe, and the heart with the pericardium, were healthy. 